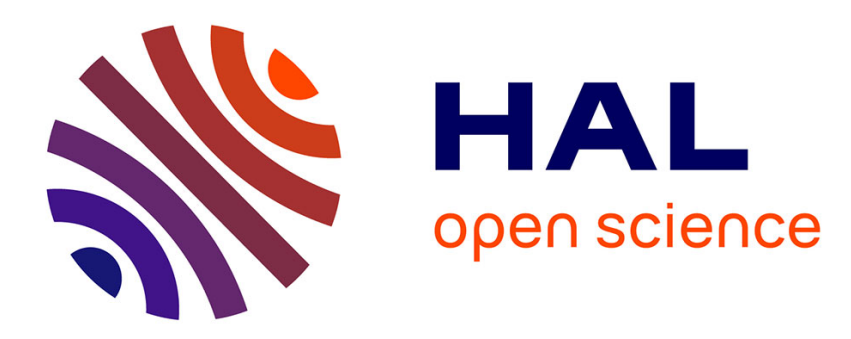

\title{
Le nouveau visage de l'État allemand
}

Olivier Giraud

\section{To cite this version:}

Olivier Giraud. Le nouveau visage de l'État allemand. Informations sociales, 2011, n 163, pp. 22-29. halshs-00658666

\section{HAL Id: halshs-00658666 \\ https://shs.hal.science/halshs-00658666}

Submitted on 10 Jan 2012

HAL is a multi-disciplinary open access archive for the deposit and dissemination of scientific research documents, whether they are published or not. The documents may come from teaching and research institutions in France or abroad, or from public or private research centers.
L'archive ouverte pluridisciplinaire HAL, est destinée au dépôt et à la diffusion de documents scientifiques de niveau recherche, publiés ou non, émanant des établissements d'enseignement et de recherche français ou étrangers, des laboratoires publics ou privés. 


\title{
Olivier Giraud, politologue
}

\begin{abstract}
Olivier Giraud est chercheur au CNRS (LISE, CNAM, Paris) en analyse comparative des politiques publiques dans les domaines des politiques de l'emploi, de la formation, du care et des relations de genre. Il s'intéresse notamment à l'analyse comparative des régimes locaux de mise en ouvre des programmes d'action publique.
\end{abstract}

\section{Résumé}

Le modèle allemand de protection sociale a été réformé de fond en comble dans les dix dernières années. L' Allemagne a longtemps su concilier un niveau élevé de protection sociale avec une forte compétitivité de son économie nationale et un système de relations professionnelles représentatif. Le bilan du régime allemand d'emploi et de protection sociale montre qu'il a donné lieu à la construction d'un régime de citoyenneté sociale inclusif et multidimensionnel. Au début des années 2000, la refondation radicale des politiques de l'emploi a représenté une rupture dans la trajectoire de ce modèle. Certaines interprétations en limitent la portée à son stricte périmètre. Selon la thèse développée dans cet article, la séquence des réformes du système de protection sociale allemand remonte aux années 90 et dépasse le champ des politiques de l'emploi. A partir de cette période en effet, les discours publics se sont focalisés sur la nécessaire baisse du coût de la protection sociale et progressivement, sur l'objectif d'activation des chômeurs. Le consensus dégagé sur ces buts a exclu du jeu politique les acteurs qui n'y adhéraient pas pleinement tels l'aile gauche du SPD et certains syndicats de branche. La rupture radicale au sein du modèle social allemand bouleverse les structures socioéconomiques du pays et pose la question de la refondation de son régime de citoyenneté sociale.

Les représentations que nous avons de l'Allemagne sont dépassées. Notre voisin n'est plus le havre de ce modèle social célébré à la fois pour l'efficacité de son économie, la modération de ses inégalités sociales et de revenus, les bonnes performances globales de sa protection sociale ou encore le fonctionnement équilibré de son système de relations professionnelles. L'Allemagne qui s'installe dans la deuxième décennie du $21^{\text {ème }}$ siècle est le pays de l'Union européenne dans lequel la pauvreté laborieuse a le plus progressé ces dernières années jusqu'à rejoindre le taux américain (Bosch \& Weinkopf 2008). Depuis 10 ans, la progression des coûts salariaux allemands a été inférieure de moitié à la moyenne des pays membres de l'Union (Destatis 2010) alors même que la désaffection à l'égard des organisations et des normes du système de négociations collectives se poursuit dans le pays. De manière concomitante à ces évolutions survenues pour l'essentiel sur le marché du travail, la RFA s'est engagée dès le milieu des années 1990, mais de façon renforcée au tournant du siècle, dans un programme impressionnant de réformes de la protection sociale. Désigné par ses promoteurs de la coalition sociale-démocrate et verte au pouvoir entre 1998 et 2005 d' « Agenda 2010 », ce train de réformes couvre un éventail important d'enjeux, des retraites, 
à l'éducation, en passant par la santé, ou surtout, les politiques de l'emploi et les politiques familiales. L'agenda 2010 décidé par le gouvernement Schröder a souvent été compris comme une rupture forte, dont les conséquences politiques furent manifestes : éclatement du parti social-démocrate, émergence d'un parti à la gauche de ce dernier - die Linke -, rupture entre le SPD et une bonne partie du mouvement syndical, série de défaites électorales pour la gauche allemande. Face à ces constats élémentaires, trois réflexions méritent d'être posées.

La première réflexion porte sur les liens entre emploi, revenu et protection sociale et brosse le portrait du «modèle allemand» tel qu'il s'était développé jusqu'à l'orée du $21^{\text {ème }}$ siècle.

La deuxième concerne l'inscription temporelle et, par-là même la logique de fond des réformes. Au-delà de la question du rythme soutenu des changements politiques, quelle a été la séquence politique, sociale ou encore économique qui a donné lieu à la transition esquissée ici à grands traits ?

Enfin, nos réflexions conclusives dresseront un bilan synthétique des conséquences économiques et sociales de la profonde transformation du domaine de l'emploi et de la protection sociale en Allemagne. Au plan politique, la refondation du modèle allemand dans ces domaines clé suppose sans doute une redéfinition parallèle du régime allemand d'intégration sociale.

\section{Régime d'emploi et de protection sociale}

Les enjeux de l'emploi, du revenu et de la protection sociale impliquent une pluralité d'arènes de régulation en partie autonomes les unes par rapport aux autres : les politiques de l'emploi, fiscales et du revenu, le domaine des relations professionnelles, le champ des politiques familiales et de la conciliation comptent parmi les plus importants à ce propos. Cependant, ces enjeux de régulation sont fortement interdépendants, à la fois dans les discours sociaux et politiques, mais aussi dans l'orientation des politiques conduites. Ainsi, la nature du système d'indemnisation du chômage (forfait minimal ou assurance-chômage proportionnelle au revenu antérieur), l'absence ou la présence d'un salaire minimum, la capacité du système de relations professionnelles à définir une norme salariale ou encore le niveau des allocations familiales et parentales agissent tous sur le revenu des actifs (Gazier \& Lechevalier 2008). Dans les différents pays, des équilibres spécifiques se forment et se maintiennent souvent durablement. Au Royaume-Uni par exemple, la faiblesse du système d'indemnisation du chômage, le caractère libéral des régulations de l'emploi et le niveau bas du salaire minimum ont conféré une place centrale au crédit d'impôt, dont la prime pour l'emploi française est inspirée : un tiers des salariés qui ont des enfants bénéficient dans ce pays de cette aide d'Etat (Clasen 2009).

Dans le cas allemand, le régime d'emploi et de protection sociale possédait jusque dans les années 1990 des contours clairs. La définition des salaires et du temps de travail relevait de la compétence de négociations entre patronats et syndicats organisés dans le contexte de branches relativement autonomes les unes par rapport aux autres ce qui autorisait le maintien d'inégalités fluctuantes entre les secteurs d'activité en fonction des évolutions marchandes. L'absence de salaire minimum était compensée par les efforts syndicaux d'homogénéisation des normes de revenu, mais aussi par une situation du marché du travail plutôt favorable aux salariés, au moins jusqu'à la réunification. La protection sociale dans le champ de l'emploi était organisée via un système d'assurance-chômage proportionnel au revenu antérieur, et aux durées et taux d'indemnisation d'assez haut niveau par rapport à d'autres pays. Au premier 
délai cadre d'indemnisation succédait une mesure d'assistance-chômage, financée sur le budget fédéral, d'un niveau moins élevé, mais dont le délai cadre était particulièrement long. Une ultime mesure de protection était l'aide sociale financée et mise en œuvre par les communes, seul de ces dispositifs à ne plus ouvrir de droits à bénéficier des mesures actives pour l'emploi, axées sur la formation ou la requalification et les emplois aidés. Le service public de l'emploi prenait la forme d'une agence fédérale contrôlée paritairement par les associations d'employeurs et les syndicats en charge des régulations de l'emploi. La loi sur la promotion du travail (Arbeitsförderungsgesetz) de 1969 avait pour but explicite non seulement de maintenir un niveau élevé d'emploi, mais aussi d'assurer la croissance de la qualité et de la qualification des emplois. En vertu de ce principe, les chômeurs avaient la possibilité de refuser tout emploi dont le salaire ou la qualification aurait été inférieure au dernier poste occupé. Priorité était de plus donnée aux mesures de formation.

Ce régime d'emploi et de protection sociale, progressivement développé depuis l'après-guerre, est arrivé à maturité dans les années 1970 et 1980. Il correspond à un modèle de citoyenneté sociale multidimensionnelle construite à la fois à travers l'appartenance à une profession par la socialisation en vertu du système de formation professionnelle, à une entreprise privilégiant les relations stables avec les salariés, à un syndicat, à une branche et enfin à une communauté solidaire à fondement professionnel, mais dont le fonctionnement est garanti par l'Etat.

\section{Temporalité et sens des réformes du régime d'emploi et de protection sociale}

Les objectifs de plein emploi et d'optimisation des postes et des qualifications au centre des dispositifs pour l'emploi jusque dans les années 1990 semblent aujourd'hui relever d'une époque révolue. Des interprétations divergentes s'efforcent d'expliquer le bouleversement récent du régime allemand d'emploi et de protection sociale.

\section{Le rôle central du politique dans les réformes}

En premier lieu, pour certains auteurs, cette dynamique est la conséquence de l'incapacité des syndicats allemands à avoir accepté la flexibilisation de l'emploi et un durcissement négocié des conditions d'accès aux prestations du système de protection sociale dans les années 1990. Cet échec du canal corporatiste de gouvernance du régime d'emploi et de protection sociale aurait alors mécaniquement conduit à imposer des réformes par la voie politique dans le but d'obtenir des résultats similaires (Palier \& Thelen 2010). Pour d'autres, l'analyse doit être centrée d'emblée sur les acteurs politiques qui ont jugé que l'accélération des déséquilibres financiers des comptes de la protection sociale au long des années 1990 est devenue insupportable à la fin de cette décennie. Les contributions sociales ont atteint le seuil symbolique de 40\% des coûts salariaux en 1996 (Streeck, Trampusch 2005) et les transferts importants du budget fédéral au comblement des déséquilibres des régimes d'assurance retraite, chômage et maladie expliquent l'essentiel du déficit budgétaire et de l'endettement cumulé des finances fédérales (Streeck 2009 ; Hinrichs, 2010). La coalition rouge-verte au pouvoir aurait dû alors s'attaquer de front à cette menace émergeante.

Ces différentes analyses mettent en scène des ruptures intervenues dans un temps ramassé et sont centrées sur l'agenda des réformes des politiques gouvernementales. Dans le domaine des politiques de l'emploi, les lois Hartz ${ }^{1}$ sont en effet emblématiques d'une reprise en main par l'Etat au détriment des formes conventionnelles de la concertation sociale avec les associations patronales et syndicales. La révélation en 2001 des mauvaises performances

\footnotetext{
${ }^{1}$ Voir article de Brigitte Lestrade dans ce numéro.
} 
de l'ancien service public de l'emploi (Bundesanstalt für Arbeit) et notamment du faible rendement des dépenses publiques dans le domaine du placement (Vermittlungsskandal) comme dans celui des mesures actives, monté en épingle par le gouvernement fédéral (Schmid 2006, p. 369), a facilité l'éviction de ces acteurs traditionnels.

Au plan des contenus, les lois Hartz ont réorganisé le service public de l'emploi selon les principes de la nouvelle gestion publique, favorisé les instruments de placement rapide des chômeurs au détriment des politiques actives traditionnelles, durci les contraintes du placement des chômeurs, assuré la promotion de contrats de travail faiblement rémunérés et non soumis à contributions sociales (mini et midi jobs cf supra), etc. (Giraud \& Lechevalier 2009). Au plan de l'indemnisation, les lois Hartz ont ramené à 12 mois la durée d'indemnisation par l'assurance-chômage, fusionné l'assistance-chômage proportionnelle dont bénéficiaient les chômeurs de longue durée et l'aide sociale au profit d'une prestation forfaitaire basse (ALG 2) qui prend l'allure d'un filet social minimal qui a consacré l'usage dans les débats du terme de Grundsicherung utilisé aujourd'hui dans tous les domaines de la protection sociale ${ }^{2}$.

Le dispositif Hartz a cependant introduit deux mesures censées compenser l'aggravation des clivages entre catégories de demandeurs d'emploi. En premier lieu, en fusionnant assistance-chômage et aide sociale, elles ont accordé à tous les chômeurs indemnisés la possibilité de bénéficier des mesures actives pour l'emploi. En second lieu, la prestation forfaitaire fonctionne également comme un complément de revenu pour les salariés dont les rémunérations sur le marché du travail sont trop basses. Cette situation est fréquente en Allemagne où il n'y a pas de salaire minimum interprofessionnel et où l'essentiel des créations d'emplois récentes s'est concentré sur des emplois à temps partiel dans des secteurs faiblement rémunérateurs ou encore sur des emplois aidés dont les rémunérations sont inférieures à 400 euros (mini-jobs) ou vont jusqu'à 800 euros (midi-jobs ${ }^{3}$ ).

\section{L'activation des politiques d'emploi et la mutation de la norme d'emploi}

En dehors de ces analyses centrées sur le rôle du politique et sur les politiques de l'emploi au sens strict, une troisième série d'interprétations analyse une séquence temporelle plus large et porte sur le rôle des changements de discours dans le domaine englobant du régime d'emploi et de protection sociale. Selon ces interprétations, les lois Hartz en tant que réforme radicale des politiques de l'emploi ne sont que l'aboutissement d'un processus de conversion d'un grand nombre des acteurs du régime de l'emploi et de la protection sociale à de nouvelles conceptions à propos des enjeux économiques et sociaux. Dès le début des années 1990, immédiatement après la réunification, le gouvernement de Helmut Kohl a lancé un grand débat national qui portait sur la place de l'Allemagne et de son système productif, dans l'économie mondialisée. Conduit sur plusieurs années, ce débat a eu pour effet d'imposer un certain nombre de représentations dont la plus importante dressait le constat que les coûts salariaux allemands comptaient parmi les plus élevés au monde et qu'il fallait enrayer leur progression de toute urgence.

Cette conclusion saillante a été alimentée et renforcée par les expertises académiques des années 1990 et 2000 insistant sur la non viabilité des régimes continentaux de protection sociale basés sur l'assurance salariale, notamment en raison de leur incapacité à favoriser la croissance de l'emploi dans les services à la personne (Scharpf \& Schmidt 2001), et par les discours diffusés à partir des arènes internationales (OCDE et, progressivement, Union

\footnotetext{
${ }^{2}$ Voir l'article d'Arnaud Lechevalier dans ce numéro.
}

\footnotetext{
${ }^{3}$ Données statistiques de la Bundesagentur für Arbeit :

http://www.pub.arbeitsamt.de/hst/services/statistik/detail/b.html
} 
Européenne). Ces dernières ont fortement mis en avant "l'activation » des politiques de l'emploi et ont organisé la focalisation des débats sur la seule norme des taux d'emploi. Le terme de " chômage » a pratiquement disparu des discours pour laisser la place à la notion ambivalente «d'activité » qui évoque à la fois les avantages des mesures actives (formation, aide au placement, emploi aidé, etc.) sur les seules dépenses d'indemnisation, la priorité donnée à la réintégration rapide sur le marché du travail, mais aussi la suspicion systématique à l'égard de chômeurs, dont "l'inactivité » pose problème. La coalition rouge-verte est arrivée au pouvoir avec un agenda clair de réforme du régime d'emploi et de protection sociale. La proximité affichée par le chancelier Schröder dans le "papier », manifeste de la troisième voie, signé avec Tony Blair (Blair \& Schröder 1999) s'est transformée en une influence directe du modèle britannique sur le processus de réforme en Allemagne (Clasen 2009)(Seeleib-Kaiser \& Fleckenstein 2007). Alors même que les modèles anglo-saxons avaient été célébrés tout au long de la décennie 1990 précisément pour la forte croissance de leurs taux d'emplois.

Le paradigme dominant dans le domaine de l'emploi à partir des années 90 n'est ainsi pas seulement centré sur la question des coûts de la protection sociale, mais il inclut également une analyse de la nécessaire activation des chômeurs. Ce paradigme dominant, une fois installé, devait durablement exclure l'ensemble des positions politiques ou revendications sociales qui s'en éloignaient trop. La gauche politique s'est divisée entre partisans et opposants à cette position jusqu'à déboucher sur la scission de l'aile gauche du parti socialdémocrate. Le mouvement syndical a lui aussi été frappé par des ruptures. Les syndicats traditionnellement les plus conservateurs, IG-BCE, fondé à partir du syndicat de la chimie, ont rejoint la position dominante, alors qu'au contraire, le syndicat Ver.di, formé de la fusion des syndicats de branche des transports, des médias, de la fonction publique, et de l'industrie des services, a évolué dans le sens d'un refus radical du processus Hartz et s'est rapproché du parti die Linke (Streeck 2009). Cette division idéologique, qui suit les initiatives de l'IGMetall des années 1980 et 1990 pour les 35 heures, puis pour la retraite à 60 ans, a contribué à affaiblir la centrale interprofessionnelle DGB, devenue pratiquement inaudible dans les débats publics.

Précisément, le régime allemand d'emploi et de protection sociale s'est également transformé en vertu des bouleversements qui ont affecté les relations professionnelles en Allemagne. Entre 1992 et 2003, le taux d'organisation des salariés a chuté de 31,3 à 19,7\% ; dans la branche emblématique de la métallurgie, le taux d'adhésion des entreprises aux associations d'employeurs s'élevait à 42,8\% en 1993 mais a reculé de 20 points en dix ans puisqu'il n'atteint plus que $22,5 \%$ en 2003 , ce qui représente cependant encore plus de la moitié des salariés du secteur (Streeck 2009). Cet affaiblissement des organisations s'est accompagné de sévères tensions entre les différentes filières de représentation du monde patronal. La désunion syndicale, la désaffection des salariés et des entreprises et enfin les pressions à la modération salariale émanant des firmes ont débouché sur trois tendances majeures depuis une quinzaine d'années. Tout d'abord, les dérogations quant aux salaires ou au temps de travail se sont banalisées dans la plupart des secteurs. Ensuite, les normes d'emploi se sont différenciées entre secteurs, ou en fonction de la taille des entreprises, notamment au détriment des salariés du secteur des services. Enfin, les syndicats se sont engagés dans la voix d'une modération salariale sans équivalent en Europe, du milieu des années 1990 jusqu'à aujourd'hui. 


\section{Vers un modèle dualisé de protection sociale?}

Les discours qui se sont imposés dans le régime d'emploi et de la protection sociale en Allemagne ont non seulement formaté les stratégies politiques dans le strict domaine des politiques de l'emploi, mais ils ont également exercé une influence forte sur les acteurs des relations professionnelles. L'affaiblissement des normes de la protection sociale s'est ainsi accompagné d'une dislocation des solidarités intersyndicales et des normes collectives négociées.

Le tournant de l'activation des politiques de l'emploi s'est fait au prix des mesures de formation et qualification dont le nombre a été divisé par trois, au profit de mesures d'incitation à des activités indépendantes entre 1998 et 2007. Sur la même période, entre 2000 et 2009, l'Allemagne a perdu plus de 2,4 millions d'emplois réguliers à plein temps, pour voir progresser à hauteur de près de 1,7 millions d'emplois à temps partiel, ou encore 1,1 millions d'emplois faiblement rémunérateurs (mini-, midi-jobs et autres emplois aidés ${ }^{4}$ ). Le poids des temps partiels concerne particulièrement les femmes et explique presque exclusivement l'accroissement de leur présence dans l'emploi. Ces évolutions entraînent un accroissement exponentiel des inégalités de revenus sur le marché du travail, une augmentation drastique du taux de pauvreté laborieuse, ainsi qu'une augmentation de la pauvreté des ménages (Mason, Salverda, 2009) qui tendent à rapprocher de plus en plus clairement la situation allemande de celle du Royaume-Uni et des Etats-Unis.

L'ensemble de ces évolutions du régime allemand d'emploi et de protection sociale invite à reposer la question de la citoyenneté sociale qui se dessine outre-Rhin. L'éclatement de la norme d'emploi qui émerge du fonctionnement du marché du travail, l'affaiblissement des affiliations syndicales et de leurs solidarités, la désaffection grandissante des entreprises à l'égard des régulations conventionnelles, la dualisation des formes de la protection sociale dans le domaine de l'emploi sont autant d'éléments qui marquent l'effacement progressif de la citoyenneté sociale multidimensionnelle à l'allemande, dont les capacités d'intégration étaient réelles. Le modèle de substitution qui émerge de cette période de transition n'est pas véritablement formulé. Le marché et la responsabilité individuelle sont cependant clairement les deux références qui gagnent de l'importance depuis une quinzaine d'années dans les débats publics. En cela, la mutation radicale du modèle social allemand déplace fortement le centre gravité d'un hypothétique modèle social européen en direction des modèles anglosaxons.

\section{Références bibliographiques :}

Blair, T. \& Schröder, G., 1999. GLASNOST Berlin - Schröder-Blair-Papier: Der Weg nach Vorne für Europas Sozialdemokraten - 08.06.1999. Available at: http://www.glasnost.de/pol/schroederblair.html [Zugegriffen Juni 8, 2010].

Bosch, G. \& Weinkopf, C., 2008. Low-wage work in Germany, New York, NY: Russell Sage Foundation.

\footnotetext{
${ }^{4}$ Statistiques de la Bundesagentur für Arbeit : http://www.pub.arbeitsamt.de/hst/services/statistik/detail/b.html
} 
Clasen, J., 2009. Les nouvelles politiques de l'emploi au Royaume-Uni et en Allemagne. Critique internationale, 43(2), 37.

Gazier, B. \& Lechevalier, A., 2008. The European Employment Strategy, Macroeconomic Policies, Institutional Regimes, and Transitional Labour Markets. In The European Social Model. Law and Policy of Transitional Labour Markets. Londres: Ashgate, S. 107-124.

Giraud, O. \& Lechevalier, A., 2009. Les réformes Hartz des politiques de l'emploi : instrument ou reflet de la normalisation du marché du travail. In Radioscopies de l'Allemagne 2009. Travaux et recherches de l'IFRI (1991), ISSN 1257-9254. Paris: Institut français des relations internationales, S. 37-56.

Mason, G., Salverda, W. (2009) 'Low pay. Living standards and employment', in: J. Schmitt et al. (2009), Low-Wage Work in Wealthy Countries, Russel Sage, New York.

Palier, B. \& Thelen, K., 2010. Institutionalizing Dualism: Complementarities and Change in France and Germany. Politics Society, 38(1), 119-148.

Scharpf, F. \& Schmidt, V., 2001. Welfare and Work in the Open Economy - From Vulnerability to Competitiveness Volume I, Oxford: Oxford University Press.

Schmid, G., 2006. Der Mensch denkt und die Institution lenkt: Zur Reformfähigkeit von Staat und Gesellschaft am Beispiel der deutschen Arbeitsmarktpolitik. Politische Vierteljahresschrift, 47(3), 367-379.

Seeleib-Kaiser, M. \& Fleckenstein, T., 2007. Discourse, Learning and Welfare State Change: The Case of German Labour Market Reforms. Social Policy \& Administration, 41(5), 427-448.

Streeck, W., 2009. Re-forming capitalism: institutional change in the German political economy. Oxford, Oxford University Press. 